\title{
Quantification of Mouse Embryonic Eye Development with Optical Coherence Tomography In Utero
}

\author{
N. Sudheendran ${ }^{1}$, M. Mashiatulla ${ }^{1}$, R. Raghunathan ${ }^{1}$, S.H. Syed ${ }^{2}$, M.E. Dickinson ${ }^{2}$, I.V. Larina ${ }^{2}$, \\ K.V. Larin ${ }^{1,2,3^{*}}$ \\ ${ }^{1}$ Department of Biomedical Engineering, University of Houston, 2028 SERC Building, Houston, TX 77204, USA \\ ${ }^{2}$ Department Molecular Physiology and Biophysics, Baylor College of Medicine, Houston, TX 77030, USA \\ ${ }^{3}$ Institute of Optics and Biophotonics, Saratov State University, Saratov, 410012, Russia \\ *e-mail: klarin@uh.edu
}

\begin{abstract}
Mouse models are commonly used as research tools to understand regulatory pathways affected by human diseases and disorders. Live imaging tools for visualization of mouse embryonic ocular tissues would be beneficial in research associated with developmental ocular defects. In this study, in utero quantitative assessment of ocular structures in mouse embryos was performed with a swept-source optical coherence tomography (SSOCT). To define developmental changes in eye morphology in live embryos, the volume of the embryonic eye lens and the globe at different embryonic stages ranging from E13.5 to E18.5 was quantified. It is determined that the major axis diameter of the eye lens and the globe was found to increase from $0.44 \pm 0.18 \mathrm{~mm}$ to $0.98 \pm 0.05 \mathrm{~mm}$ and from $0.56 \pm 0.22 \mathrm{~mm}$ to $1.23 \pm 0.14 \mathrm{~mm}$, respectively, as the embryo ages from E13.5 to E18.5. For the same stages, the volume of the eye lens and globe was found to increase from $0.028 \pm 0.027 \mathrm{~mm}^{3}$ to $0.32 \pm 0.08 \mathrm{~mm}^{3}$ and from $0.09 \pm 0.08 \mathrm{~mm}^{3}$ to $0.75 \pm 0.27 \mathrm{~mm}^{3}$, respectively. These results suggest that OCT can accurately assess developmental processes of ocular structures and can be potentially used to assess embryonic ocular growth in mouse mutants with eye abnormalities and to study the effect of toxicological and pharmacological agents. (C) 2015 Samara State Aerospace University (SSAU).
\end{abstract}

Keywords: Eye volume; in utero; mouse embryo; optical coherence tomography.

Paper \#2039 received 2015.01.13; revised manuscript received 2015.02.25; accepted for publication 2015.02.26; published online 2015.03.28.

\section{References}

1. J. V. Gross, and B. D. Perkins, "Zebrafish mutants as models for congenital ocular disorders in humans," Mol Reprod Dev 75, 547-555 (2008).

2. N. Goodall, et al., "3-Dimensional modelling of chick embryo eye development and growth using high resolution magnetic resonance imaging," Exp Eye Res 89, 511-521 (2009).

3. Y. Ai, et al., "A mouse model of galactose-induced cataracts," Hum Mol Genet 9, 1821-1827 (2000).

4. M. G. Anderson, et al., "Mutations in genes encoding melanosomal proteins cause pigmentary glaucoma in DBA/2J mice," Nat Genet, 30, 81-85 (2002).

5. J. J. Windle, et al., "Retinoblastoma in transgenic mice," Nature 343, 665-669 (1990).

6. N. A. Syed, et al., "Transgenic mice with pigmented intraocular tumors: tissue of origin and treatment," Invest Ophthalmol Vis Sci. 39, 2800-2805 (1998).

7. P. Parasoglou, et al., "High-resolution MRI of early-stage mouse embryos," NMR Biomed, (2012).

8. S. W. John, et al., "Essential iris atrophy, pigment dispersion, and glaucoma in DBA/2J mice," Investigative ophthalmology \& visual science 39, 951-62 (1998).

9. F. S. Foster, et al., "In vivo imaging of embryonic development in the mouse eye by ultrasound biomicroscopy," Investigative ophthalmology \& visual science 44, 2361-2366 (2003). 
10. A. S. Brown, et al., "Quantitation of hemodynamic function during developmental vascular regression in the mouse eye," Investigative ophthalmology \& visual science 46, 2231-7 (2005).

11. D. Huang, et al., "Optical coherence tomography," Science 254, 1178-1181 (1991).

12. I. V. Larina, et al., "Live imaging of blood flow in mammalian embryos using Doppler swept-source optical coherence tomography," J Biomed Opt. 13, 060506 (2008).

13. I. V. Larina, et al., "Hemodynamic measurements from individual blood cells in early mammalian embryos with Doppler swept source OCT," Opt. Lett. 34, 986-988 (2009).

14. N. Sudheendran, et al., "Speckle variance OCT imaging of the vasculature in live mammalian embryos," Laser Phys Lett. 8, 247-252 (2011).

15. I. V. Larina, et al., "Sequential Turning Acquisition and Reconstruction (STAR) method for four-dimensional imaging of cyclically moving structures," Biomed Opt Express 3, 650-660 (2012).

16. S. H. Syed, et al., "Optical coherence tomography for high-resolution imaging of mouse development in utero," Journal of biomedical optics 16, 046004 (2011).

17. I. V. Larina, et al., "Optical coherence tomography for live phenotypic analysis of embryonic ocular structures in mouse models," Journal of biomedical optics 17, 081410 (2012).

18. V. Tuchin, Tissue Optics - Light Scattering Methods and Instruments for Medical Diagnosis, 2nd Edition, SPIE Press, Bellingham, WA (2007).

19. T. Ramaesh, et al., "Corneal abnormalities in Pax6(+/-) small eye mice mimic human aniridia-related keratopathy," Investigative ophthalmology \& visual science 44, 1871-1878 (2003).

20. E. Steingrimsson, et al., "Molecular-Basis of Mouse Microphthalmia (Mi) Mutations Helps Explain Their Developmental and Phenotypic Consequences," Nat Genet 8, 256-263 (1994).

21. C. Harch, H. B. Chase and N. I. Gonsalves, "Studies on an Anophthalmic Strain of Mice .6. Lens and Cup Interaction," Dev Biol. 63, 352-357 (1978).

\section{Introduction}

Embryonic development is an intricate process regulated by both, genetic and environmental factors. Animals such as zebra fish [1], chick, [2] and mice [3] are commonly used as models for human ocular diseases. Mice have been the preferred animal to study mammalian development and human congenital disorders due to easily manipulated genomes. A wide range of mutant models are available for various human diseases, including ocular defects such as cataract [3], glaucoma [4], retinoblastoma, [5] and intraocular tumors [6].

Histological studies have been traditionally used to study ocular defects; however, the lack of 3D information, tissue deformation during fixation and processing, and the inability to perform live studies limits the information that can be gained and precludes longitudinal analysis of a single sample. Imaging tools such as magnetic resonance imaging (MRI) and ultrasound (US) allow performing repeated observations in mouse embryos. These methods are noninvasive and can capture real-time, 3D, structural and dynamic information, however, the spatial resolution of these approaches is a limitation for embryonic ocular research. While ex vivo MRI imaging of fixed mouse embryos has previously been performed with up to 20 $\mu \mathrm{m}$ of resolution, in utero imaging only allows for resolution of about $100 \mu \mathrm{m}$; besides with long imaging times $(\sim 2 \mathrm{~h})$ required to achieve sufficient signal-tonoise ratio (SNR) [7]. High-frequency US is a wellestablished approach for mouse embryo research. Measurements of the anterior chamber using an ultrasound bio-microscope (UBM) were performed to study the development of glaucoma in mutant mice at different ages ranging from 2 to 30 months [8]. UBM has also been utilized to monitor the development of ocular tissues at different stages of embryonic and postnatal development, for instance to examine the regression of hyaloid vasculature in mice $[9,10]$. High frequency ultrasound imaging can achieve axial resolution of up to $30 \mu \mathrm{m}$ and lateral resolution of about $90 \mu \mathrm{m}$, which might not be sufficient to resolve fine details within the developing eye.

Recently we have demonstrated that Optical Coherence Tomography (OCT) technique has clear advantages in resolution and the provides sufficient contrast for structural and functional imaging of mammalian embryos [12-15]. OCT is a noninvasive optical imaging modality which is capable of high speed ( $\mathrm{MHz}$ A-line rate), high resolution $(\sim 2-20 \mu \mathrm{m})$, realtime 3-D imaging without requirement for any external contrast agents as it relies on inherent optical contrast [11]. By combining live mouse embryo manipulation approaches with OCT, we developed approaches for live in vivo and in utero longitudinal mouse embryonic imaging and demonstrated that these methods allow for visualization of ocular structures $[16,17]$. In this study, we performed, for the first time to the best of our knowledge, a quantitative assessment of mouse embryonic ocular structures at different stages of development in utero with OCT. Acquired measurements were correlated with previously reported values obtained with UBM. While our results correlate with previously reported values, the spatial resolution of OCT gives a potential for more accurate assessment of embryonic development and visualization of structures, which are not accessible with other available methods. 


\section{Methods}

\subsection{Imaging system}

A swept-source optical OCT (SS-SOCT) system has been employed in this study to visualize embryonic ocular structures [14]. The SS-OCT system comprises of a swept-source laser (Thorlabs SL-1325-P16), with a wavelength range of $1325 \pm 50 \mathrm{~nm}$, scan rate of $16 \mathrm{kHz}$ and an output power of $12 \mathrm{~mW}$. The power on the sample arm is $3.6 \mathrm{~mW}$. A beam-splitter splits the laser beam in the ratio of $90 \%$ and $10 \%$ into the sample and reference arms, respectively. The interference pattern formed from the back-reflected light from the sample and the reference arms is detected by a balanced photodetector (Thorlabs, PDB 140C) and digitized by a 14-bit ADC (AlazarTech). An external Mach-Zehnder interferometer (MZI) clock is used for linear k-space calibration. Fast Fourier transform (FFT) is performed on the calibrated data to obtain 1D depth scan (A-scan). Galvanometer-mounted mirrors are used to perform 2D and $3 \mathrm{D}$ imaging by scanning the laser beam on the surface of the sample. The axial and transverse resolutions of the system are measured to be $12 \mu \mathrm{m}$ (in air) and $15 \mu \mathrm{m}$, respectively.

\subsection{Mouse manipulations}

All animal manipulation procedures described here were approved by the Animal Care and Use Committee of the University of Houston. The animal manipulation protocol is described in details in our previous publication [16]. Briefly, the pregnant CD-1 mice at the desired gestinational stage have been anesthetized using isoflurane by inhalation. The anesthetized mouse was placed on a heating platform maintained at $37^{\circ} \mathrm{C}$ during the entire procedure. An electrical razor was used to remove the abdominal fur at the incision site. An incision of $\sim 1-2 \mathrm{~cm}$ was made on the abdomen to expose the uterine horn for imaging. OCT imaging of the embryonic ocular structure was performed through the uterine wall. From every litter, 2-3 embryos were imaged. Each 3D volume contained $512 \times 512 \times 512$ pixels. The lateral scan size depended on the size of the eye. The later pixel resolution was ensured to be at least as much as the transverse resolution $(15 \mu \mathrm{m})$. The in depth scan range was always $3 \mathrm{~mm}$ (in air). The beam in the sample arm was focused on the ocular structures of the embryonic eye. At least five embryos from each stage were analyzed. Each embryo was imaged only once for the purpose of this study. Following the imaging session, the adult mouse was sacrificed. Measurements were made at different stages ranging from embryonic day (E) E13.5 to E18.5.

\subsection{Eye volume measurements}

To estimate the volume of the eye, we assume the eye to be of oblate spheroid shape [9]. The volume (V) of the oblate spheroid is given by equation (1); [9]:

$$
V=\frac{4}{3} \pi a^{2} c
$$

where, $\mathrm{a}$ is the major axis radius and $\mathrm{c}$ is the minor axis radius. The minor axis of the eye is along the optical axis of the eye and the major axes radii are perpendicular to it. Orienting embryos in utero for imaging is a challenging task, and the optic axes of the embryo eye can be randomly located within the acquired data set during imaging. To overcome this, a Matlab based application was developed to perform manual 3D rotation of the data set to orient the crosssectional visualization plane along the optic axis of the eye. The application allows a user to view and manually rotate the eye in three dimensions until the entire major and minor axis radii can be captured in a single 2D frame individually. Since the original scanning distances as well as the rotation angles in three dimensions are known, the distances after $3 \mathrm{D}$ rotation can be computed using coordinate transformation. The number of pixels along each direction of the $3 \mathrm{D}$ volume was scaled corresponding to the scanning distance so that the $3 \mathrm{D}$ voxel is cubic. The refractive index was assumed to be 1.4 [18] and does not change significantly with development. Once the 3D OCT data sets were rotated to correct the alignment, ImageJ software was used to measure the diameters along the major and minor axes.

\section{Results}

Typical OCT images containing embryonic eyes acquired in utero at different stages are presented in Fig. 1. Even at the latest state (E18.5), the imaging depth of OCT is able to capture the entire eye with sufficient contrast. Different embryonic ocular structures including lens and retina are easily identifiable (as labeled). The difference in size and morphology is clearly distinguishable between the developmental stages, as expected.

To orient the OCT data sets along the optic axes of the embryonic eye for measurement, we developed and applied a Matlab based application (as described in the Materials and Methods section). The effect of 3D rotation of the mouse embryo eye data set is demonstrated in Fig. 2. Figs. 2(a, c) show the OCT axial and transverse cross-sections of the eye prior to application of the code, while Figs. 2(b, d) demonstrate corresponding cross-sections after the OCT data set rotation.

The major and minor axes diameters were measured from the oriented $3 \mathrm{D}$ data sets using the ImageJ software. To estimate the volume of the eye, the shapes of the eye lens and the eye globe are assumed to be oblate spheroid. An oblate spheroid is circular in its transverse cross-section (in our convention, xy plane) and elliptical in its axial cross-sections (xz or yz plane), with its major axes ( $\mathrm{x}$ and $\mathrm{y}$ axes) along the transverse directions and its minor axis along the axial direction $(\mathrm{z}$ axis). Since an oblate spheroid is circular in its 


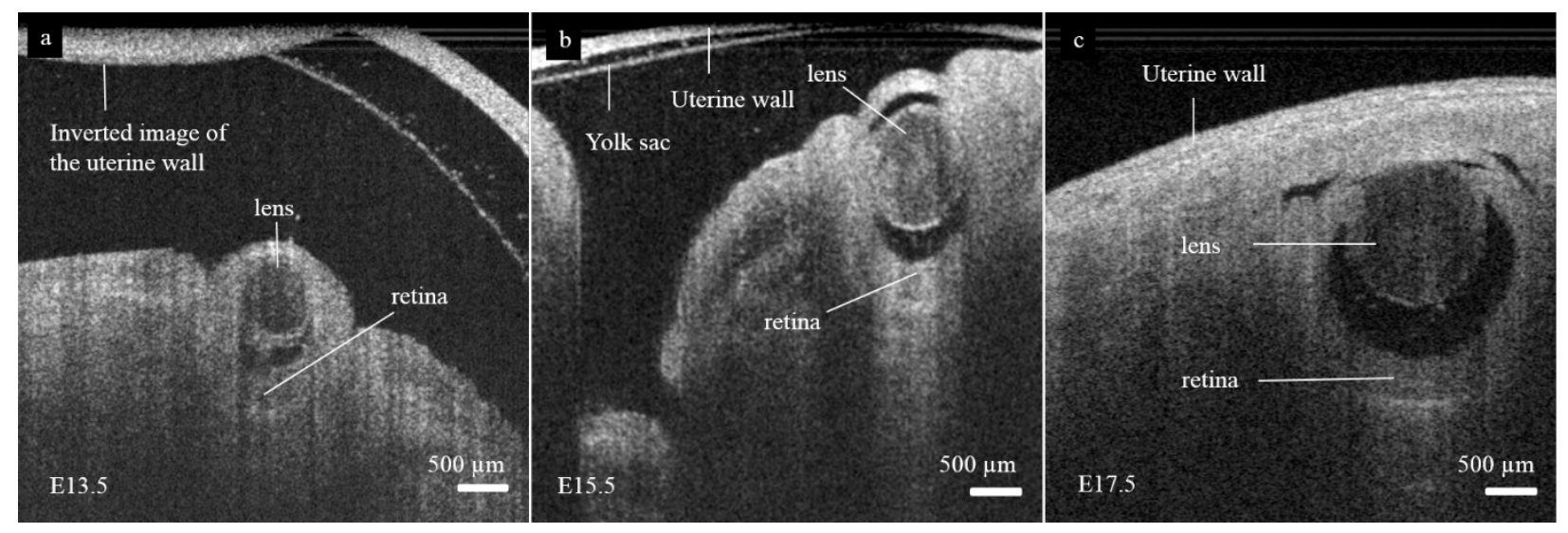

Fig. 1 2D OCT images through the eye in utero at different stages of embryonic growth.
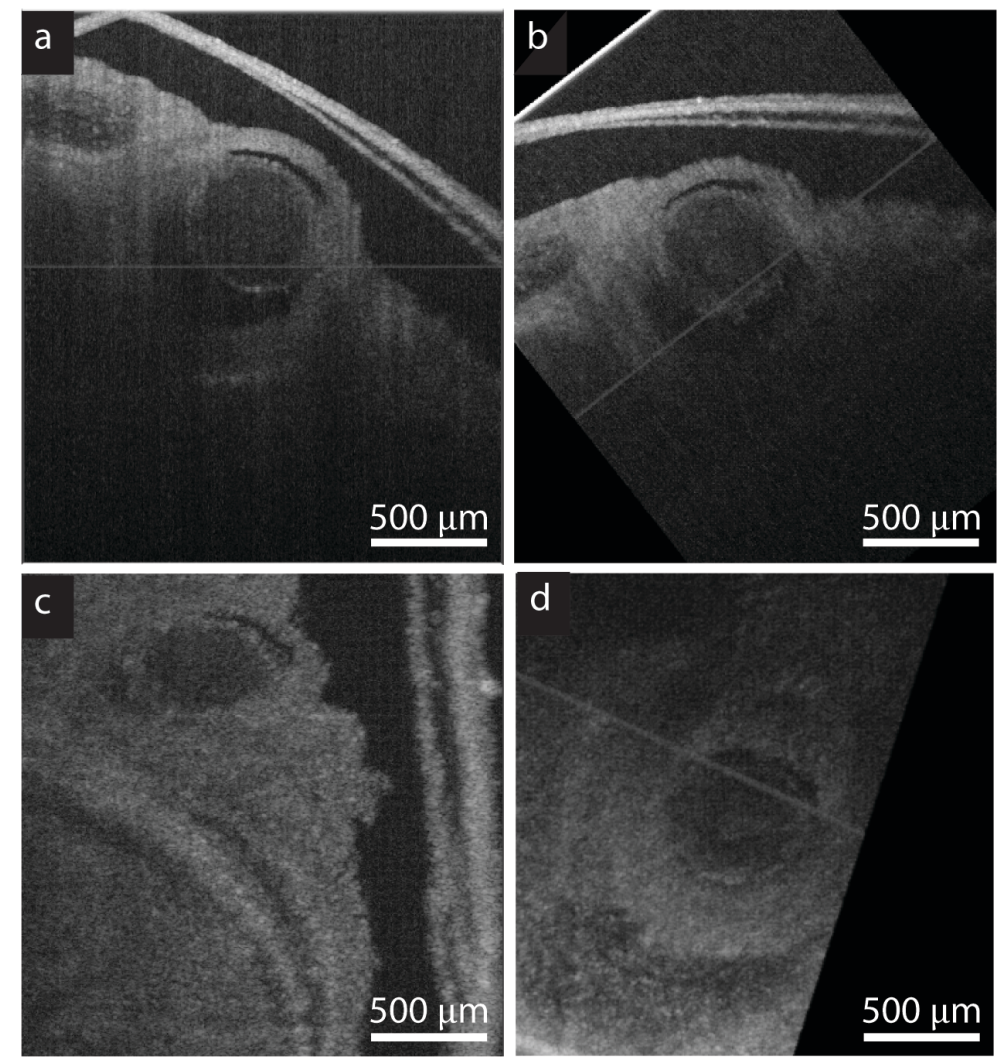

Fig. 2 2D cross-sectional OCT images before and after rotational orientation of the 3D OCT data set. (a) Axial crosssectional image before 3D rotation. (b) Axial cross-sectional image after 3D rotation. (c) Transverse cross-sectional image before application of the rotational orientation algorithm. (d) Transverse cross-sectional image after the rotational orientation of the $3 \mathrm{D}$ data set.

transverse direction, the measurement of the major axis diameters along the $\mathrm{x}$-axis and the $\mathrm{y}$-axis should be the same. It can be observed from Fig. $2 b$ that the axial cross-section of the eye lens and globe is elliptical and the circular nature of the transverse cross-section is apparent in Fig. 2d. To further validate the assumption of oblate spheroid shape, the major axis diameter from both the $\mathrm{x}$-axis and the $\mathrm{y}$-axis (transverse axes) of the eye lens and globe at different growth stages were measured and presented in Figs. $3 \mathrm{a}$ and $3 \mathrm{~b}$, respectively.
The slope of 0.91 with $\mathrm{R}^{2}$ (coefficient of determination) value of 0.84 for the eye lens and slope of 0.85 with $R^{2}$ value of 0.87 for the eye globe implies that the diameters of major axes in the transverse direction are similar, which justifies the assumption that the entire eye lens and eye globe are close to the oblate spheroidal shape [9].

The major axis diameters from different stages of embryonic growth, obtained using OCT, are presented in Fig. 4. It can be noted from Figs. $4 \mathrm{a}$ and $4 \mathrm{~b}$ (green 

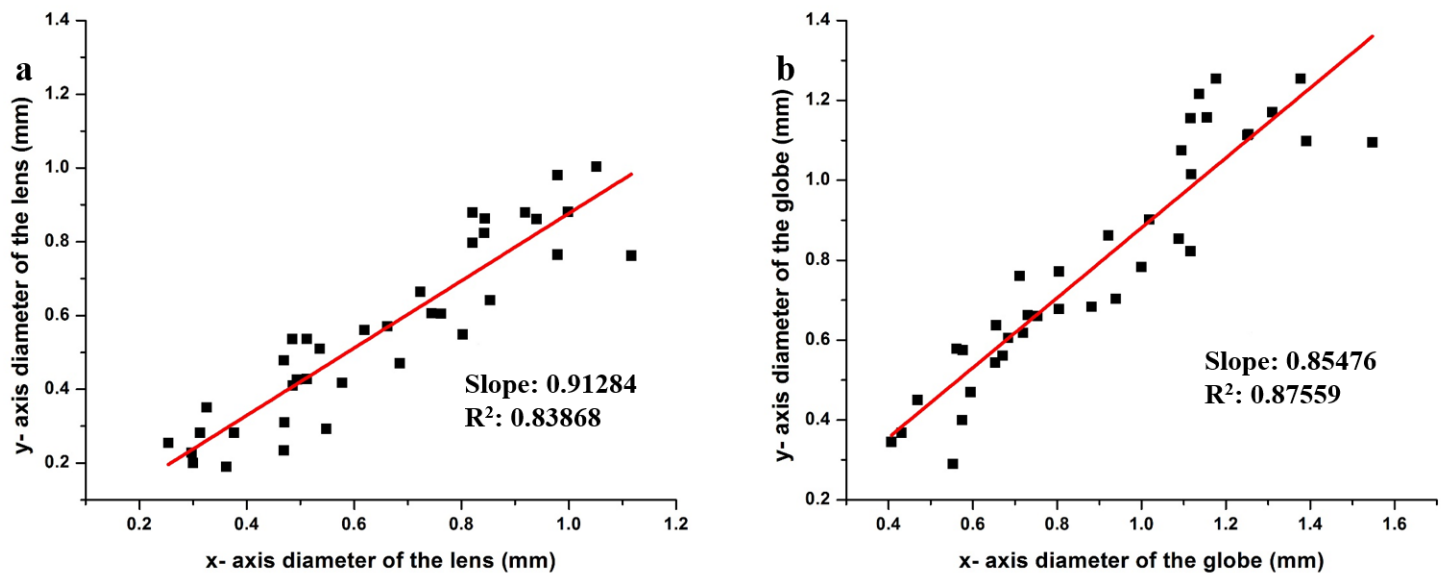

Fig. 3 Plots of major axis diameter measured along the x-axis and y-axis (transverse axes) of the eye (a) lens (b) globe.
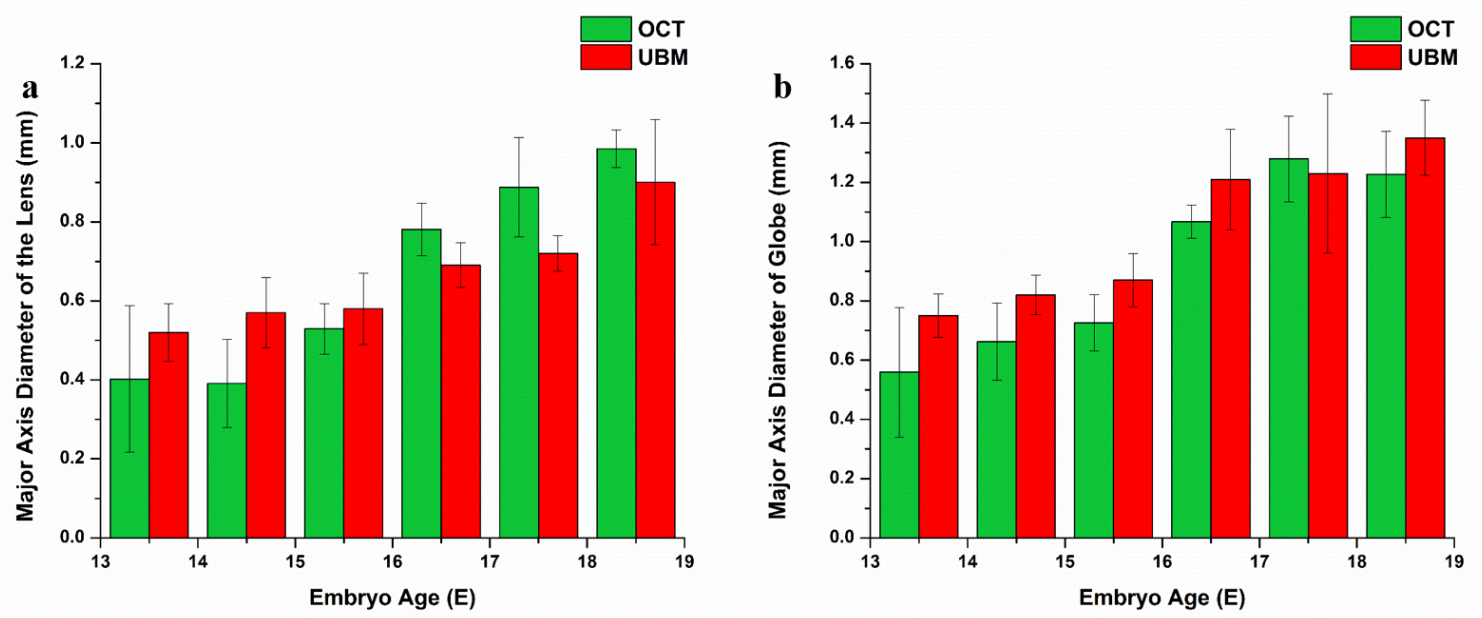

Fig. 4 Major axis diameter measurements of the eye (a) lens (b) globe correlate well with previously reported UBM values $(n \geq 5)$. The error bars represent SD. The UBM data is obtained from [9].

bars) that there is roughly a 2 -fold increase in major axis diameter of the eye lens and a 3 -fold increase in major axis diameter of the eye globe from E13.5 to E18.5. Using the measurements of the major and minor axis diameters, the volumes of the eye lens and the globe were computed using Eq. (1). These results correlate well with Foster et.al [9], where the authors measured the major axis diameters and volumes of eye lens and globe using UBM (resolution of $40 \mu \mathrm{m}$ ). The results obtained using UBM (from [9]) are presented in Fig. 4 (red bar). The major axis diameter measurements are within similar range, as compared to OCT measurements, across the different stages of development. Note that the standard deviation values from the UBM measurements are comparatively larger to the ones from OCT measurements, suggesting higher precision with the latter technique. The volume measurements of the eyes are presented in Fig. 5. It was determined that the volume of the eye lens and globe increased from $0.028 \pm 0.027 \mathrm{~mm}^{3}$ to $0.32 \pm 0.08 \mathrm{~mm}^{3}$ and from $0.09 \pm 0.08 \mathrm{~mm}^{3}$ to $0.75 \pm 0.27 \mathrm{~mm}^{3}$, respectively. All the values in Fig. 4 and 5 are reported as average \pm SD format, the SD arises due to variability between different embryos.

\section{Discussion}

As evident from structural images of the embryo eyes, the contrast and resolution offered by OCT enables clear distinction of the embryonic ocular structures in utero. The measurements of the major axis diameters of lens and globe using OCT between E13.5 to E18.5 are within similar range as the measurements from previous study using UBM [9]. UBM has an inferior resolution $(\sim 40 \times 60 \mu \mathrm{m})$ as compared to OCT $(\sim 12 \times 15 \mu \mathrm{m})$. Moreover, the major and minor axis diameters were measured without any correction for the tilt of the eye. This can result in underestimating the actual diameters. It can also be noted from [9], that the reported values for the volumes of the eye lens and the globe is not 
consistent with the respective values of the major axis diameters, as the authors likely used different measurements to obtain the final values.

Although OCT has the advantage of superior resolution and contrast compared to UBM, limited depth penetration in tissues is a significant drawback. This limitation requires the use of surgery to expose the uterus of the pregnant mouse to image the embryos. However, our group has devised protocols to perform in utero longitudinal studies of mouse embryos. We have also demonstrated that this methodology does not affect the growth of the embryos [16].

\section{Conclusions}

In this preliminary study, we have used measurements of eye lens and globe volumes as means to demonstrate ability of OCT to study ocular development in mouse embryos. The volumes of the eye lens and the globe have been shown to increase from $0.028 \pm 0.027 \mathrm{~mm}^{3}$ to $0.32 \pm 0.08 \mathrm{~mm}^{3}$ and from $0.09 \pm 0.08 \mathrm{~mm}^{3}$ to $0.75 \pm 0.27$ $\mathrm{mm}^{3}$, respectively, from E13.5 to E18.5. This study suggests that our approach can potentially be used for live quantitative characterization of ocular tissues in a variety of ocular research studies. These include monitoring morphological changes during eye development in mutant mice models for human ocular diseases such as aniridia [19], microphthalmia [20] and anophthalmia [21]. Our future studies will involve an automated algorithm to perform the rotation of the dataset and also longitudinal measurements of various biometrics related to the eye in both normal and mutant mouse embryos.

In conclusion, we have demonstrated that in utero OCT embryo imaging approach allows for live quantitative assessment of mouse embryonic ocular growth with higher resolution, than other currently available methods. Potentially, this approach can be used to assess embryonic ocular growth in mouse mutants with eye abnormalities and to study the effect of toxicological and pharmacological agents.

\section{Acknowledgement}

This research was supported by NIH HL077187, HL095586, HL120140, T32 HL007676, and AHA 10SDG3830006.
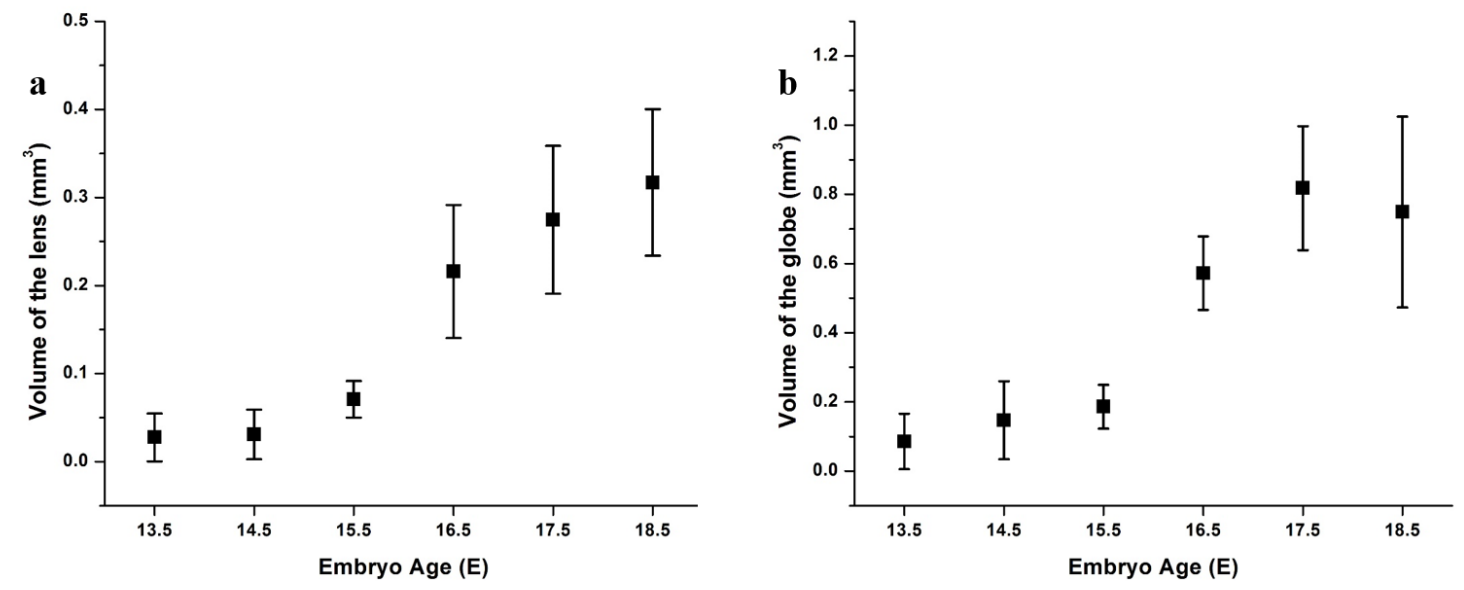

Fig. 5 Plot of volume of the eye (a) lens (b) globe measured using OCT in utero. The error bars represent SD ( $\mathrm{n} \geq 5$ ). 